\title{
Les racines de la « révolution du 25 janvier » en Égypte : une réflexion géographique
}

\section{Delphine Pagès-El Karoui et Leila Vignal}

\section{(2) OpenEdition}

\section{Journals}

\section{Édition électronique}

URL : http://journals.openedition.org/echogeo/12627

DOI : 10.4000/echogeo. 12627

ISSN : 1963-1197

\section{Éditeur}

Pôle de recherche pour l'organisation et la diffusion de l'information géographique (CNRS UMR 8586)

\section{Référence électronique}

Delphine Pagès-El Karoui et Leila Vignal, « Les racines de la « révolution du 25 janvier » en Égypte : une réflexion géographique », EchoGéo [En ligne], Sur le Vif, mis en ligne le 27 octobre 2011, consulté le 30 avril 2019. URL : http://journals.openedition.org/echogeo/12627 ; DOI : 10.4000/echogeo.12627

Ce document a été généré automatiquement le 30 avril 2019.

\section{(c) (i) (9)}

EchoGéo est mis à disposition selon les termes de la licence Creative Commons Attribution - Pas d'Utilisation Commerciale - Pas de Modification 4.0 International 


\title{
Les racines de la « révolution du 25 janvier » en Égypte : une réflexion géographique
}

\author{
Delphine Pagès-El Karoui et Leila Vignal
}

1 Hosni Moubarak, autocrate régnant sur l'Égypte depuis 30 ans, est finalement tombé, lâché par l'armée, à la suite de l'intense mobilisation pacifique de la jeunesse égyptienne galvanisée par l'exemple tunisien. La rapidité de son départ (18 jours seulement ont suffi, entre le 25 janvier et le 11 février 2011), qui faisait écho à celui de Zine el-Abidine Ben Ali, a surpris le monde. Les signes d'exaspération étaient certes présents - l'image d'un pays sous pression revenait souvent dans les conversations des Égyptiens - mais le système de coercition du régime autoritaire paraissait suffisamment fort pour briser toute menace réelle. Finalement, le système a été ébranlé, le raïs est tombé mais... l'armée a gardé les rênes du pouvoir.

2 Le déclenchement de la « révolution du 25 janvier », comme la désignent les Égyptiens, encouragé par l'exemple tunisien, a été le fait d'éléments conjoncturels (assassinat du jeune blogueur Khaled Saïd en juin 2010, élections législatives truquées en novembre, attentats contre l'Église copte d'Alexandrie en décembre) qui sont venus attiser le mécontentement et fissurer le mur de la peur sur fond d'une décennie de blocage du système politique et de promesses non tenues d'une libéralisation économique aux bénéfices peu partagés.

3 Cet article présente la chronique d'une révolte en gestation, mais il l'articule à l'analyse des évolutions structurelles qui ont transformé en profondeur la société égyptienne au cours des quarante dernières années. Ces racines de la « révolution du 25 janvier » sont de trois ordres: démographique (baisse de la fécondité); sociétal (essor de l'alphabétisation et diffusion de l'éducation) (Courbage, Todd, 2007); spatial, avec une urbanisation massive qui a vu l'Égypte passer en quelques décennies d'un pays à dominante rurale à un pays dont plus de $80 \%$ de la population vit en milieu urbain ${ }^{1}$ (Moriconi-Ebrard, 2011). Or, c'est la conjugaison de ces trois évolutions majeures qui 
explique les modalités spatiales de la révolution égyptienne : une révolution des villes, et qui passe par une réappropriation de l'espace public, bien au-delà l'emblématique place Tahrir au centre du Caire.

\section{Retour sur la révolution du 25 janvier}

4 Pour les Égyptiens, la révolution, thawra, est en marche, même si le système n'a pas été totalement renversé. De même, elle n'a pas commencé avec le départ de Hosni Moubarak, le 11 février, mais avec la première manifestation d'envergure, le 25 janvier 2011. La « révolution de la peur ", la rupture fondamentale, se situe donc en amont de la chute du président haï, dans l'élan contestataire par lequel les Égyptiens ont réussi à faire entendre leur voix et à recouvrer leur dignité en s'attaquant ouvertement au système autoritaire et corrompu.

\section{Trois semaines historiques}

\section{Un soulèvement populaire et national}

5 Le mouvement de contestation a démarré le mardi 25 janvier: plusieurs blogueurs militants ont lancé sur les réseaux sociaux un appel à manifester « contre la torture, la pauvreté, la corruption et le chômage » lors d'une " Journée de la colère » (chaque grande mobilisation porte un nom). Bien que cette manifestation ait été interdite par les autorités, plus de 90000 personnes avaient annoncé sur Facebook leur intention de manifester. Le 25 janvier, premier fait remarquable, le nombre total de manifestants était estimé à plus de 20000 personnes: cette mobilisation tranchait nettement avec la faiblesse numérique habituelle des manifestations aux mots d'ordre politiques des années 2000, qui ne rassemblaient au mieux que quelques centaines de manifestants vite contrôlés par les services de sécurité et de police. Deuxième fait remarquable, les cortèges se sont formés un peu partout en Égypte. Certes, les manifestants ont été les plus nombreux au Caire, mégapole de 15 millions d'habitants. Environ 15000 personnes ont convergé vers la place Tahrir à partir de plusieurs quartiers du Caire - des quartiers populaires comme Shûbra, Bûlaq, Imbâbâ ou des quartiers de classes moyennes et supérieures comme Mohandissîn. Simultanément, des manifestations ont eu lieu dans de nombreuses villes, partout en province : à Alexandrie, la seconde ville du pays, mais aussi dans le Delta, en Haute-Égypte, dans la région du Canal de Suez et dans le nord de la péninsule du Sinaï. Dès le départ, les manifestations du 25 janvier opèrent donc une double rupture par rapport aux mobilisations de la décennie 2000 : on observe à la fois un saut quantitatif (plus grand nombre de manifestants) et géographique puisque les cortèges sont présents sur l'ensemble du territoire national.

6 Le vendredi 28 janvier, jour de congé hebdomadaire, le mouvement s'amplifie. Au Caire, à la sortie de la grande prière, des cortèges très fournis investissent le centre-ville et rejoignent la place Tahrir, qui devient l'épicentre de la contestation. Les slogans en appellent à la fin de l'état d'urgence en place depuis 30 ans, à la mise en place d'élections libres, au départ du raïs. Le pont Qasr al-Nil, qui mène à la place Tahrir, est alors le théâtre de scènes inédites, inouies : la foule désarmée fait reculer les canons à eau de la police. 
7 Le régime réagit de deux façons. D'une part, pour jouer sur l'inquiétude des classes moyennes, il fait ouvrir les prisons et libérer des prisonniers de droit commun. Il fait également appel à des baltagi-s, hommes de main à la solde du pouvoir, à la fois pour contrer les manifestants et pour s'attaquer aux biens privés. Dès le 25 janvier, la mobilisation est sonnée. Policiers et membres de services de sécurité en civil sont envoyés pour attaquer les manifestants et procéder à de très nombreuses arrestations. Le régime met en place un couvre-feu (non respecté), fait appel à l'armée pour maintenir l'ordre, bloque les communications (Internet, téléphonie mobile et voies terrestres). Des contremanifestations de soutien à Hosni Moubarak sont organisées, composées de partisans "spontanés » ou "encadrés"-souvent des hommes pauvres, payés à la journée et amenés de province par autobus entiers. À partir du 28 janvier, après que de nombreux commissariats et véhicules des forces de l'ordre ont été incendiés, la police disparaît des rues. Pour contrer le risque d'insécurité, les habitants s'organisent en milices citoyennes de protection, quartier par quartier. Le mercredi 2 février, la foule mobilisée place Tahrir est confrontée à l'attaque d'hommes montés sur des dromadaires et des chevaux - ceux là même qui promènent sur leur dos les touristes sur le plateau des Pyramides à Giza, et dont les propriétaires vivent du tourisme, qui sont arrêtés par les manifestants. Selon Amnesty International (2011), les arrestations arbitraires et la torture furent massivement employées contre des milliers de personnes. Le bilan humain de la révolte s'élève à, au moins, 840 morts et près de 6500 blessés.

8 Pourtant, au fil des jours, le mouvement s'étend, au Caire et en province. À partir du 8 février, les grèves se multiplient dans de nombreux secteurs (transports, textile, hydrocarbures, santé, éducation...). Par ailleurs, aux manifestations quotidiennes s'ajoutent de grands rendez-vous qui rassemblent des centaines de milliers de personnes dans le pays. La "Marche du million », le mardi $1^{\text {er }}$ février, qui visait à rassembler plus d'un million de manifestants au Caire, atteint son objectif malgré les restrictions d'accès mises en place par le régime. Le basculement final a lieu entre le jeudi 10 et le vendredi 11 février. Le jeudi, l'allocution télévisée du président Moubarak, dans laquelle il annonçait son maintien à la présidence jusqu'aux élections de septembre tout en déléguant ses pouvoirs à son nouveau vice-président Omar Souleimane, est jugée insuffisante et galvanise la révolte. Le vendredi 11 février, journée de mobilisation baptisée de façon prémonitoire «Jour de l'adieu », voit le départ du raïs et le transfert du pouvoir à l'armée, en l'occurrence la direction collégiale de son Conseil suprême.

\section{Le divorce entre Moubarak et l'armée}

9 Face à la contestation, le régime donna d'abord des signes d'intransigeance - le premier discours de Hosni Moubarak, le 28 janvier, ne céda rien. Mais, dès le samedi 29, le président égyptien annonçait la nomination d'Omar Souleimane, le puissant chef des services de sécurité, comme vice-président (fonction que Hosni Moubarak n'avait pas pourvue depuis son accession au pouvoir en 1981), et le 31 janvier, un nouveau gouvernement. Hosni Moubarak s'est donc trouvé forcé de négocier, moins sous la pression directe de la rue que, face à la crise qui montait, avec les autres composantes du régime, et en particulier la puissante armée égyptienne. La rupture entre Hosni Moubarak et l'armée fut consommée le 31 janvier, lorsque cette dernière annonça qu'elle ne tirerait pas sur le "grand peuple égyptien", dont les revendications étaient « légitimes ». En position de force, elle obtint par ailleurs l'éviction de Gamal Moubarak, le fils cadet du président, qui depuis le début des années 2000 se préparait officieusement 
à la succession de son père, projet qui n'avait jamais obtenu l'assentiment de l'institution militaire et qui avait créé de profondes tensions entre celle-ci et le raïs.

Or, l'armée est une institution puissante et autonome. Issu d'un coup d'État militaire en 1952, qui renverse la monarchie en Égypte, le régime a besoin de son soutien et de sa garantie. C'est le rôle historique de l'armée dans la construction de la nation égyptienne moderne qui lui assure soutien et légitimité au sein de la population. C'est également le résultat de sa composition sociologique: elle demeure un lieu de passage obligé du parcours des hommes du pays et l'une des rares institutions dans laquelle la perspective d'une ascension sociale est encore possible pour un jeune homme d'extraction modeste. Enfin, sa capacité d'action et d'influence passe par les réseaux que forment ses officiers supérieurs qui, une fois à la retraite, sont nommés à la tête des grandes administrations territoriales ou des entreprises nationales du pays.

11 Son rôle est donc plus que militaire : il est politique, et parfois même social. En effet, à côté de sa puissance (un effectif de 750000 hommes, un budget annuel alimenté notamment par une aide militaire américaine de plus d'un milliard de dollars par an depuis la signature des accords de Camp David en 1978), l'armée, grand propriétaire foncier, contrôle de très nombreuses entreprises dans l'agro-alimentaire, la construction, l'hôtellerie... Ainsi, l'armée est intervenue dans nombre de crises sociales ou de catastrophes qu'a connues le pays: par exemple, en 2008, lors de l'envolée des prix des denrées alimentaires, elle a mis ses réserves agricoles et ses usines au service de la fabrication et de la distribution de pain fortement subventionné.

Les dirigeants de l'armée ont donc rapidement fait le calcul de l'impossibilité de soutenir longtemps l'impopulaire président, dont le refus de prendre en compte les revendications de la rue, était destructeur pour les fondements du régime, ainsi que pour leurs propres intérêts. L'armée, véritable colonne vertébrale du régime, a donc décidé de lâcher Moubarak en promettant au peuple de se porter garant de la révolution, tout en reconstituant parallèlement l'appareil répressif, comme l'a prouvé la multiplication des procès à l'encontre des manifestants qui entendent poursuivre la révolution après le 11 février. C'est là l'une des grandes ambiguïtés de cette révolution égyptienne.

\section{Une « révolution Facebook »?}

13 Si les nouvelles technologies de communication et d'information ont joué un véritable rôle de contre-pouvoir, leur rôle mérite d'être nuancé (Béchir Ayari, 2011).

La pénétration de ces nouvelles technologies est récente et rapide en Égypte. En ce qui concerne Internet, une décennie a suffit pour faire passer les chiffres d'usagers de 450000 en 2000 à 23,5 millions en janvier 2011, soit un taux de pénétration de $30 \%$ de la population totale ${ }^{2}$. Facebook comptait 6,8 millions d'utilisateurs en avril 2011 - dont $70 \%$ de jeunes -, chiffre en très forte hausse depuis le début de l'année (ils n'étaient que 4,5 millions fin décembre) ${ }^{3}$. La chaîne satellitaire Al-Jazira, très regardée en Égypte, a été la première à diffuser des films réalisés sur des portables qui circulaient déjà sur le web, sa couverture de la révolution égyptienne étant clairement pro-manifestants. Plus massif encore, du fait de l'investissement financier et du niveau de formation moindres qu'elle nécessite, est le succès de la téléphonie mobile qui est venu compenser la mauvaise couverture de téléphonie fixe : en janvier 2011, on comptait 71 millions d'abonnements (taux de pénétration de plus de $90 \%$ de la population, contre $12 \%$ pour les lignes fixes). 

efficacité dans la diffusion de mots d'ordre et leur capacité à transmettre une information non soumise au contrôle de la censure. Ce sont des médias propices à l'incubation d'une culture commune et à la création de " communautés imaginées » au sens de B. Anderson. Dans le contexte d'un régime sécuritaire répressif, elles permettent aux contestataires de se compter et ainsi de rompre l'isolement et le mur de la peur. Pendant les manifestations, les jeunes utilisaient SMS et Twitter pour se fixer des points de ralliements et éviter les barrages policiers. Ainsi, ils parvenaient à créer plusieurs foyers contestataires, toujours en mouvement. Les autorités ont très tôt saisi le rôle crucial des nouveaux médias, à tel point qu'elles ont tenté de les censurer, en ayant recours à un blocage total pendant plusieurs jours d'Internet, des lignes téléphoniques et même d'AlJazira. Puis, dans un deuxième temps, la sécurité intérieure et l'armée ont essayé d'imiter la méthode des manifestants. Aussi, de nombreux Égyptiens ont-ils reçu des SMS progouvernementaux ambigus les invitant à « démasquer les traitres et les criminels » ou du type : «Jeunesse égyptienne, méfie toi des rumeurs et écoute la voix de la raison ».

Ces nouvelles technologies ne sont pourtant pas à l'origine de la révolte. D'abord parce que les activistes égyptiens avaient déjà investi Internet pour leurs campagnes précédentes, sans rencontrer le même succès. Un appel à la grève générale le 6 avril 2008 avait été lancé par des blogueurs du Caire en soutien aux ouvriers de Mahalla, grande ville textile du Delta, via une page Facebook et avait été relayé par la téléphonie mobile : le soulèvement ne prit guère et fut rapidement réprimé. Mais un acteur majeur de la révolution de 2011 était né, le « Groupe du 6 Avril ». Ensuite, parce que la prise de parole publique critique s'est progressivement libérée en Égypte depuis 2005, avec l'émergence en particulier d'une nouvelle presse et de chaînes de télévision indépendantes. Enfin, parce que la réussite de la mobilisation de 2011 a été le fait d'un mouvement dans lequel la diversité de la société égyptienne a été représentée : des jeunes, bien sûr, comme des générations plus âgées; des femmes comme des hommes; des classes moyennes comme des classes populaires; des employés comme des ouvriers, des commerçants, des fonctionnaires, des professions libérales, des entrepreneurs, etc...; des habitants des grandes villes du nord comme des bourgs du sud.

17 Cette généralisation est ce qui lui a permis d'atteindre la masse critique nécessaire à la fragilisation du régime. La révolte a été le fait de citoyens qui ont à la fois créé et investi, physiquement et politiquement, un espace public inédit - car largement inexistant comme tel au cours des décennies précédentes. Cet espace politique public est urbain: c'est dans la ville et par la ville que la volonté collective s'est incarnée. Les réseaux sociaux ont accéléré ce phénomène, ils ne l'ont pas créé.

\section{Les villes aux avant-postes : une révolution urbaine}

18 En dépit du rôle central joué par Le Caire et plus précisément par le centre symbolique de la révolution, la place Tahrir, des manifestations importantes ont eu lieu en province.

Alexandrie, deuxième métropole du pays avec 4,3 millions d'habitants, a été très active dans les mobilisations. Quasiment toutes les capitales de gouvernorats ont été touchées par des manifestations, sans compter les bastions industriels qui n'ont pas ce rang administratif, comme Mahalla ou Kafr al-Dawwâr, qui sont seulement chefs-lieux de district $^{4}$ (cf. illustration 1). Lieux par excellence de l'exercice du pouvoir local, principaux centres de services, les capitales de gouvernorats abritent notamment les universités. En

EchoGéo, Sur le Vif | 2011 
2011, les villes ont été le lieu principal des mobilisations contre le régime. Pour autant cela ne signifie pas que les manifestants sont uniquement des citadins: de nombreuses personnes se sont déplacées des villages ou des petits villes ${ }^{5}$ vers les grandes villes régionales pour venir manifester devant des bâtiments symboliques, comme le siège du gouvernorat ou de la Sécurité Centrale.

Illustration 1 - La révolution égyptienne de 2011

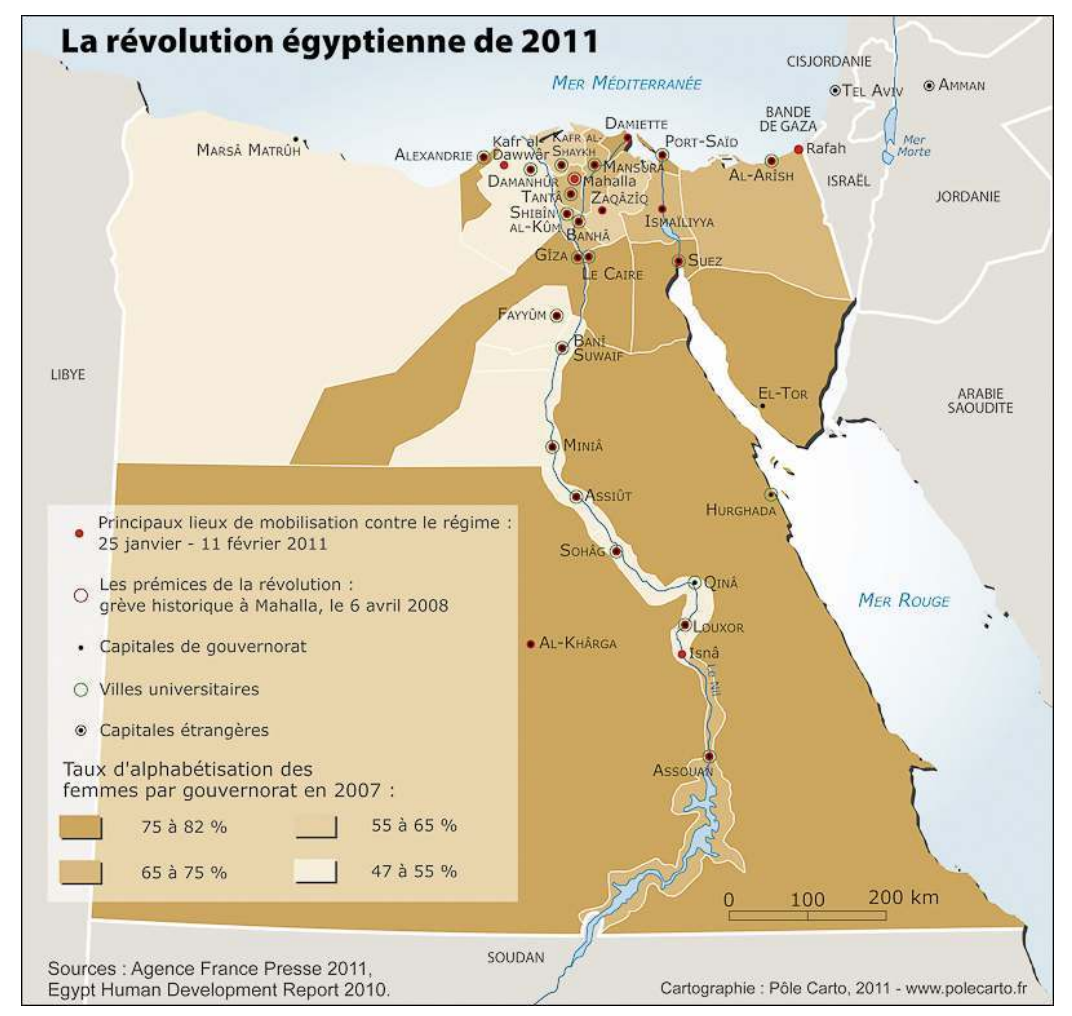

On a manifesté dans les grandes villes du Delta, Tantâ, Mahalla, Mansûra, Zaqâzîq - où les islamistes avaient remporté un grand succès en 2005 - et dans celles du Canal de Suez : les premiers morts sont tombés à Suez, grande ville industrialo-portuaire où existe une véritable tradition de révolte contre le pouvoir central (Bruyas, 2007). À l'autre extrême $\mathrm{du}$ spectre, les gouvernorats périphériques (désert occidental, Mer Rouge et Sinaï), très peu peuplés, sont restés globalement à l'écart des mobilisations, à l'exception d'actes extrêmement violents (attaques au lance-roquettes contre des bâtiments publics à alArish ou Rafah) menés par des Bédouins dans un contexte d'affrontements récurrents avec la police depuis les attentats de Taba en 2004 (Sanmartin, 2010).

En Moyenne et en Haute-Égypte, les manifestants ont investi les grands axes, places et esplanades des bâtiments publics des grandes villes, principalement des capitales de gouvernorat. Cependant, la Vallée du Nil n'a pas été à la pointe de la contestation - alors qu'en Tunisie, ce sont précisément des régions les plus dépourvues, les plus à l'écart de la mondialisation, celles de l'intérieur, qu'est née la contestation (Daoud, 2011). Pourquoi la Haute-Égypte, plus pauvre, moins équipée, moins connectée, n'a pas joué un rôle déclencheur similaire dans la contestation à celui joué par l'intérieur de la Tunisie. Faut-il y voir la crainte des habitants, éprouvés par les dures années de la répression des islamistes dans les années 1990 ? Ou bien faut-il évoquer un meilleur contrôle du territoire par les forces de police, du fait des troubles terroristes fondamentalistes des 
années 1990 (surtout en Moyenne Égypte) ? Ou peut-on encore en conclure à la priorité pour le gouvernement de préserver à tout prix des troubles les hauts-lieux du tourisme international (Haute Égypte, entre Louxor et Assouan) et donc d'y déployer l'appareil sécuritaire nécessaire?

\section{La place Tahrir : la réappropriation de l'espace public}

Si les premiers jours, les mobilisations ont eu lieu dans plusieurs quartiers de la capitale, notamment dans les zones informelles qui regroupent $60 \%$ des habitants du Grand Caire, la "prise de la place Tahrir» par la foule et sa centralité au cours des semaines qui suivent est exemplaire de l'extrême centralisation politique et administrative du pays.

Vaste esplanade située au centre de l'agglomération, la place Tahrir constitue un véritable cœur de l'histoire de la ville, à la fois politique, intellectuel et culturel. Cette place participe en effet de la mémoire politique de la ville comme centre du Caire « moderne » érigé à la fin du XIXe siècle qui est désigné sous le terme de "centre-ville » ( wast al balad), et dont l'image fut longtemps associée à la société cosmopolite qui s'est développée sous la domination coloniale. Après l'incendie de 1952, qui détruisit les hautslieux de ce centre moderne, la place devint le symbole de la révolution de 1952; la nouvelle république égyptienne se la réappropria en la rebaptisant place de la «Libération » (tahrîr). Sur la place, ou à proximité, se trouvent de grandes institutions politiques et administratives (siège du PND ; Ministère de l'Intérieur ; Parlement ; Banque Centrale; Mugamma, un immense bâtiment offert par l'URSS, qui concentre de nombreuses administrations; diverses branches de ministères; siège de la télévision nationale) ou culturelles (Université Américaine du Caire, Musée archéologique, etc...).

Récemment, certaines de ces institutions ont été relocalisées, ou le seront bientôt, dans les nouveaux quartiers qui s'érigent en périphérie de l'agglomération. Ce vaste mouvement de délocalisation, planifié par les pouvoirs publics, vide le centre-ville d'une partie de son histoire, de ses fonctions politiques, intellectuelles (départ de l'Université américaine dans les nouveaux quartiers de New Cairo), et touristiques (départ annoncé du Musée égyptien sur le plateau des Pyramides). Il met donc en scène la banalisation du centre-ville du Caire, « quartier (presque) comme un autre » dans une immense ville de 15 millions d'habitants au sein de laquelle diverses centralités commerciales, industrielles, tertiaires, résidentielles rivalisent. Or, en investissant physiquement et politiquement la place Tahrir, les manifestants du 25 janvier ont rendu compte de la persistance politique et symbolique de certains lieux et de la mémoire urbaine - en l'occurrence celle d'une "libération" trop rapidement confisquée. Au sein des campements de fortune qui ont couvert la place pendant trois semaines, la « République de Tahrir » affirme que le peuple est au centre de la nation comme il est au cœur de l'urbain. Conquérir l'espace public et l'émancipation politique nécessite en quelque sorte de passer par la réappropriation / libération de la ville.

\section{La montée des contestations}

La «République de Tahrir » s'affirme aussi comme le lieu d'une utopie urbaine de mixité sociale et politique, où l'ensemble de la société (et pas seulement ses classes moyennes) se retrouve et se redécouvre telle qu'elle est. Ainsi, beaucoup des "martyrs" de la révolution morts sur la place Tahrir ont été identifiés comme venant des quartiers 
populaires (qui sont pour la plupart informels). Car la révolution du 25 janvier est notamment l'expression d'une société confrontée à un double blocage, économique et politique. Celui-ci est d'autant plus frustrant que la politique de libéralisation engagée dans les années 1990 s'est révélée être ce que Eberhart Kienle désigne comme "une grande illusion» (Kienle, 2001): la libéralisation annoncée n'a conduit ni à une libéralisation économique réelle et partagée, ni à une libéralisation politique.

\section{Les promesses non tenues de la libéralisation}

Au début des années 1970, face à la faillite du modèle dirigiste et planifié d'inspiration socialiste dans lequel l'État et le secteur public jouaient un rôle majeur, Anouar al-Sadate avait annoncé l'ouverture économique (infitâh). Mais l'Égypte n'a véritablement changé de paradigme économique qu'en 1991, lorsque le pays, acculé par une dette faramineuse, a été contraint de signer un plan d'ajustement structurel avec le FMI et la Banque mondiale.

Cette libéralisation économique s'est traduite par un désengagement de l'État via un programme de privatisations, une politique d'ouverture commerciale (baisse des barrières tarifaires via des accords commerciaux internationaux), des mesures de modernisation de l'appareil bancaire et législatif de soutien au secteur privé légal et d'encouragement des investissements directs étrangers (IDE). C'est principalement avec le gouvernement d'Ahmad Nazif (2004-janvier 2011) que ce programme s'est accéléré. Les taux de croissance annuelle qui accompagnent ce changement de paradigme économique sont flatteurs - plus de $5 \%$ en moyenne malgré les crises conjoncturelles (crise asiatique à la fin des années 1990, crise financière mondiale à partir de 2008). Les autres indicateurs macro-économiques sont également florissants : après une incertaine décennie 1990, les IDE atteignent plus de 6,7 milliards de $\$$ en $2009^{6}$, l'activité touristique explose (près de 14 millions de visiteurs en 2009-1010 pour des revenus directs estimés à 11,6 milliards de \$7), le commerce international, quoique modeste, croît (près de 73 milliards en 2009/2010, avec une balance commerciale à l'équilibre $\left.{ }^{8}\right)$.

La géographie de l'Égypte en est transformée par bien des aspects (Pagès, Vignal, 1998) : de nouvelles industries tournées vers la production de biens de consommation, issues à la fois de capitaux autochtones et internationaux, s'installent dans les zones industrielles des villes nouvelles; une société de consommation se développe incarnée par les hypermarchés et shopping malls qui distribuent massivement ces produits ainsi que des biens importés en plein essor; la croissance du secteur touristique révolutionne les rivages de l'Égypte encore désertiques il y a vingt ans (Vignal, 2010a), la manne ouverte par l'État à la promotion immobilière privée dans les périphéries désertiques du Grand Caire conduit à un extraordinaire étalement urbain, où rivalisent villes et compounds de luxe destinés aux classes aisées (Denis, Vignal, 2003). Mais la libéralisation économique a aussi d'autres conséquences : les classes populaires et moyennes voient leurs conditions de vie se détériorer sous le poids du désengagement de l'État de ses missions de service public, du chômage, de l'inflation, de la pauvreté - 22 \% d'Égyptiens vivent sous le seuil de pauvreté ${ }^{-}$, du coût et de la pénibilité de la mobilité....

Plus globalement, les résultats macro-économiques ne se traduisent pas en termes de développement réel pour au moins quatre grandes catégories de raisons: d'une part, comme le soulignent les économistes Françoise Clément et Jean-Yves Moisseron (2007), les bons résultats macro-économiques sont en partie alimentés par des revenus de type 
rentier (revenus du Canal de Suez et des hydrocarbures, transferts financiers des travailleurs égyptiens émigrés, tourisme). D’autre part, la croissance macro-économique crée peu d'emplois, en particulier dans les secteurs qui ont attiré la majorité des nouveaux investissements (banques, services, industries de biens de consommation), tandis que le blocage du marché du travail s'est traduit par la croissance exponentielle du secteur informel au cours de la décennie 2000 (neuf millions de personnes en 2006 ${ }^{10}$ ). Troisième raison, le décalage profond entre les plans de modernisation du cadre légal, administratif et technique et la réalité de leur mise en œuvre (Vignal, 2010b). Ce décalage renvoie, en quatrième lieu, à l'économie politique du pays. Si les rapprochements entre la sphère économique et les cadres politiques ont existé avant le règne de Moubarak, la proximité des milieux politiques et économiques n'a jamais été aussi forte que depuis une vingtaine d'années. Les hommes d'affaires proches du régime ont bénéficié de protections, d'octrois préférentiels et non transparents de licences et de prébendes, d'accès privilégiés aux opportunités offertes par les privatisations. Un tel rapprochement des intérêts d'une oligarchie économique et de l'oligarchie politique, s'illustre par le rôle proéminent acquis au sein du PND par Ahmad Ezz, magnat de l'acier, qui s'est enrichi en instaurant un quasi-monopole sur cette matière première indispensable. Devenu bras droit de Gamal Moubarak, Ahmad Ezz est d'ailleurs l'une des premières cibles des slogans des manifestants. Dès le 17 février, il est mis en détention provisoire pour s'expliquer sur l'obtention douteuse de permis de construire industriels (Denis, 2011). Ces pratiques à grande échelle de corruption, de clientélisme, de favoritisme et d'accaparement de biens publics ont été vivement conspuées par les manifestants de la révolution du 25 janvier et sont au cœur des procès des élites économiques et politiques qui se multiplient depuis l'été 2011.

\section{Le blocage politique}

30 Hosni Moubarak accède à la présidence en 1981 à la suite de l'assassinat du successeur de Nasser, Anouar al-Sadate, trente ans après la « révolution des officiers libres » de 1952, coup d'État militaire qui destitua la monarchie constitutionnelle du roi Farouk. La loi sur l'état d'urgence est depuis 1981, reconduite indéfiniment sous des prétextes divers par le régime, qui brandit notamment, dans les années 1990, la menace d'attentats islamistes. Cette loi interdit les manifestations et autorise les arrestations et les détentions sans procès. Les pratiques de torture se sont multipliées, notamment au détriment des Frères musulmans, sous la houlette de services de sécurité intérieure tout puissants. Les représentants du peuple au Parlement sont élus au cours d'élections truquées par le régime. Le Parti National Démocrate (PND) domine la chambre, et les partis autorisés sont sans pouvoir; ils secondent de fait le PND et ne forment pas une réelle opposition. Un contrat implicite est passé entre le régime et les Frères musulmans, principale force d'opposition, bien que non officiellement reconnue: le régime a toute liberté dans le domaine politique, tandis que le contrôle du champ social, via la réislamisation des mœurs, est laissé aux Frères musulmans et à leurs organisations caritatives. Le 25 janvier 2011, les Frères reçurent le mot d'ordre du gouvernement de ne pas participer aux manifestations.

31 Pour satisfaire la demande américaine, premier pourvoyeur d'aide du pays, un simulacre d'ouverture politique a eu lieu en 2005. Les élections législatives ont été moins contrôlées : les candidats dits indépendants, issus des Frères musulmans, ont remporté, 
fait sans précédent, 88 sièges, pour seulement 11 sièges pour l'ensemble des partis d'opposition. L'élection présidentielle était officiellement ouverte à d'autres candidats mais le seul candidat à se présenter contre Hosni Moubarak, Ayman Nour, ayant remporté $7 \%$ des voix, fut bientôt arrêté sous des prétextes fallacieux. La fin de règne annoncée d'un président bientôt octogénaire accélère la fermeture politique tandis que Hosni Moubarak prépare son fils cadet Gamal à sa succession. En 2002, Gamal Moubarak est propulsé $n^{\circ} 2$ du PND. Proche des milieux d'affaires, il soutient la formation du gouvernement d'Ahmad Nazif surnommé le " gouvernement des hommes d'affaires ». Celui-ci engage un programme de modernisation économique en 2004. Il est aussi accusé d'être le vecteur de la confiscation de l'appareil politique du PND au profit des milieux économiques dirigeants, ce qu'illustrerait le parcours ascensionnel d'un industriel comme Ahmad Ezz ( $c f$. supra). La mascarade des élections législatives truquées de 2010, orchestrée par Ahmad Ezz, est l'éclatante démonstration de ce blocage politique dans laquelle se trouve, plus que jamais, l'Égypte à la fin des années 2000. En novembre, les deux scrutins, perclus d'irrégularités et de fraudes, donnent naissance à un Parlement dominé par le PND, avec une opposition réduite à 15 sièges. L'ouverture politique en trompe-l'œil de 2005 a vécu.

Enfin, la confiscation de la scène politique passe également par un strict contrôle de toute contestation. Jusqu'en 2011, les seules expressions publiques contestataires tolérées par le régime portaient sur des événements se déroulant à l'extérieur: les manifestations de soutien à la deuxième intifada palestinienne en 2000 et celles contre l'occupation américaine de l'Irak. Les activistes, notamment dans le domaine des droits de l'Homme, ont vu leurs marges de manœuvre strictement contrôlées. Les mouvements alternatifs d'opposition comme Kefaya (Assez!), lancé en 2004, ne sont pas parvenus à trouver un soutien au-delà d'une petite élite intellectuelle, ses manifestations ne rassemblant que quelques centaines de personnes. Cependant, l'action de Kefaya, relayée par la presse indépendante, a gagné en visibilité et a brisé quelques tabous, notamment celui de la succession «dynastique» promise à Gamal Moubarak. Elle a aussi participé du développement progressif de modes de mobilisation inédits, en dehors des partis, dont la révolution du 25 janvier est issue.

\section{Une décennie de contestations sociales}

Le soulèvement égyptien s'inscrit dans la continuité d'une décennie marquée par de nombreuses contestations sociales, en rupture avec l'image d'une société civile apathique souvent véhiculée par les médias occidentaux. Contestations ouvrières d'abord, qui n'ont cessé de s'amplifier sous différentes formes : grèves, occupations d'usines, manifestations. Selon Joel Beinin (2011), plus de deux millions d'ouvriers ont mené plus de 3500 actions collectives au cours des années 1998-2010. Plus de 111 actions sont recensées pour le seul mois d'avril 2010 dans tous les gouvernorats du pays, même si la concentration sur le Caire, premier pôle d'emploi industriel du pays, reste forte (Ben Néfissa, 2010). Ces mobilisations, souvent corporatistes, se sont pour certaines émancipées des syndicats existants, totalement affiliés au pouvoir, en créant des structures autonomes (l'Union Générale Indépendante des Travailleurs de l'Autorité de la Taxe Foncière en 2008 et l'Union générale des Techniciens de Santé en décembre 2010 et, en 2011, après le départ de Moubarak, l'Union Indépendante de l'Autorité des Transports Publics du Caire, qui représente 60000 travailleurs). Face à cette intense agitation sociale, le gouvernement a 
lâché du lest, en augmentant en novembre 2010 le salaire minimum mensuel à 400 livres égyptiennes par mois, soit un triplement des seuils précédents (Beinin, 2011).

Plus encore, au cours des années 2000, des catégories auparavant peu engagées sont venues se joindre à ces mobilisations «traditionnelles » portées par les ouvriers, comme les hauts fonctionnaires de l'État, les professeurs d'université, les experts du ministère de la justice, les fonctionnaires des impôts (Ben Néfissa, 2009). La contestation touche aussi les quartiers informels, dont les habitants manifestent fréquemment contre l'absence de services publics ou contre les décisions de destruction ou d'expropriation de leurs logements. Dans les zones rurales, les raisons de la colère sont tout aussi nombreuses : absence ou mauvaise qualité de l'eau potable, problème du partage de l'eau d'irrigation, expropriations et augmentation des loyers de la terre....

L'exaspération prend une dimension plus ouverte et généralisée en 2008 avec la flambée des prix alimentaires. Plus de quinze personnes meurent dans des affrontements devant des boulangeries vendant du pain subventionné. L'armée ouvre ses stocks et fait tourner ses boulangeries, et les salaires des fonctionnaires sont augmentés de $30 \%$. La grande grève organisée alors par les ouvriers des usines textiles publiques de Mahalla, grande ville mono-industrielle du Delta et grand centre du syndicalisme ouvrier égyptien (PagèsEl Karoui, 2008), fait la une des journaux. Un groupe de jeunes blogueurs du Caire crée une page Facebook qui invite à lancer une grève générale en soutien aux ouvriers de Mahalla : le "Mouvement du 6 avril », qui appellera à la mobilisation le 25 janvier 2011, est né. Il appelle à deux manifestations de soutien, au Caire et à Alexandrie, qui ne seront guère suivies. Des affrontements violents ont lieu à Mahalla, où des slogans ouvertement hostiles à Moubarak sont lancés. La répression est féroce (3 personnes tuées et 250 arrêtées).

Le mouvement du 25 janvier est donc le fruit d'une fusion entre des mobilisations politiques nouvelles, dans leurs formes comme dans leurs revendications, et de mobilisations sociales qui ont été autant de lieux d'apprentissage pour des militants de plus en plus chevronnés. Emerge ainsi le tableau d'un soulèvement qui s'étend bien audelà d'une mobilisation de classes moyennes privilégiées et mondialisées.

\section{Les révolutions silencieuses de la société égyptienne}

La chute de Moubarak a été déclenchée par une mobilisation pacifique mais obstinée qui s'est réalisée en dehors de tout programme, idéologie ou leader clairement affirmés. C'est une révolution "du peuple», du bas vers le haut, qui «invente une autre dynamique politique où l'ordre n'est pas préconstruit, mais en situation de se construire et d'évoluer au fil des événements » (Badie, 2011).

38 En atteste de façon remarquable la rapidité avec laquelle, pendant la révolte, se sont mises en place les milices citoyennes et les mots d'ordre de responsabilité, qui est représentative de la capacité d'auto-organisation de la société égyptienne. Le retrait de l'État d'une partie de ses missions collectives, la bureaucratisation à outrance et la corruption qui est présente à tous les niveaux de l'administration et de l'espace public, ont en effet depuis longtemps conduit les Égyptiens à trouver d'autres types de ressources pour essayer, tant bien que mal, d'agir, d'entreprendre, de contourner un système qui rend très difficile, voire impossible, l'initiative individuelle. La prolifération du système informel/illégal dans tous les domaines de la vie l'illustre bien. En 2011, le 
rejet qui s'est exprimé est, de ce point de vue, non seulement celui d'un système qui ne fonctionne pas, mais aussi (et peut-être surtout), d'un système qui empêche.

La révolution rend ainsi compte des changements profonds d'une société égyptienne qui a connu de profondes transformations sociologiques, anthropologiques et spatiales au cours des quarante dernières années. Trois « racines » du 25 janvier, trois révolutions d'ordre sociologique et sociétal, nous semblent particulièrement importantes: une révolution démographique, une révolution de l'alphabétisation, une révolution urbaine (et des mobilités).

\section{Une révolution démographique et anthropologique}

Forte de 77 millions d'habitants, l'Égypte est en train d'achever sa transition démographique : la fécondité est tombée de 6 enfants par femme en 1960 à 3 en 2010. Cette baisse de la fécondité est à mettre en relation avec le niveau d'éducation de la population et plus particulièrement, l'alphabétisation. En 2006, $71 \%$ de la population sait lire et écrire.

41 Certes, la baisse de la fécondité, tout comme la diffusion de l'alphabétisation, s'inscrit dans le cadre de profonds clivages spatiaux. La chute de la fécondité a, en effet, commencé dans les grandes villes avant de se propager au monde rural. Mais, en 2008, l'écart de fécondité entre monde urbain et rural s'est considérablement réduit (respectivement 2,7 contre 3,2 enfants par femme). De plus, on constate un alignement des comportements démographiques entre d'une part, les gouvernorats urbains ${ }^{11}$ (Le Caire, Alexandrie et les villes du Canal de Suez) et l'urbain dans le Delta, avec un taux identique de 2,6 enfants par femme, et d'autre part, avec le rural du Delta et l'urbain de la Vallée ( 3 enfants par femme). Cette convergence souligne par contraste le « retard » du rural de la Haute-Égypte, avec 3,6 enfants par femme (Ambrosetti, 2011). Ces données rappellent ainsi que si le clivage urbain/rural se réduit en Égypte, le décrochage du Sa'îd (Haute-Égypte) demeure préoccupant, cette région cumulant les records nationaux de pauvreté et de sous-développement.

Ce taux d'alphabétisation global masque également les inégalités hommes/femmes, notamment dans les zones rurales, mais celles-ci sont en train de se résorber pour les classes d'âge les plus jeunes. Longtemps confinées à l'espace domestique par un système patriarcal, les femmes ont vu leur place dans la société égyptienne évoluer profondément ces dernières décennies : l'égalité pour l'accès à l'école est quasiment atteinte et, en 2008, $56 \%$ des étudiants diplômés de l'université sont des femmes. Les filles sont donc aujourd'hui plus éduquées que leurs pères... et demain que leurs maris !

Or, la conjugaison de la baisse de la fécondité et de la généralisation de l'éducation modifient les structures familiales et annoncent la fin du système patriarcal, fondé sur la soumission des frères cadets à l'aîné au sein de la fratrie et des femmes aux hommes au sein de la famille. Pour que ce système se maintienne, il faudrait en effet au moins plusieurs frères (un aîné et un cadet au minimum), ce qui n'est plus nécessairement le cas dans la famille de deux ou trois enfants (Fargues, 2000). Par ailleurs, la place des filles est transformée: d'une part parce que les fratries désormais plus petites peuvent n'être composées que de filles, et d'autre part parce que les filles sont désormais autant éduquées que les garçons, ce qui annihile l'autorité masculine auparavant conférée par la seule alphabétisation des garçons. L'égalité sociologique entre les sexes est donc en marche. Ce faisant, comme le soulignent Youssef Courbage et Emmanuel Todd (2007), 
l'effet conjugué de la transition démographique et de l'alphabétisation débouche sur une véritable révolution anthropologique, porteuse d'autonomisation des êtres et de logiques d'individualisation des parcours. Elle peut conduire à de véritables " crises de transition » qui remettent en cause les fondements de l'ordre social hérité, crises dont le contenu politique varie selon les sociétés.

\section{Une société désormais urbaine}

L'urbanisation joue un rôle essentiel dans la diffusion de ces nouveaux comportements. Avec $95 \%$ de la population qui n'occupe que $5 \%$ du territoire, l'Égypte présente un cas de densités « utiles » extrêmes ( 2200 habitants au $\mathrm{km} 2$ sur ces $5 \%$ du territoire habité) qui brouillent la distinction entre l'urbain et le rural (Pagès-El Karoui, 2008). Pourtant, l'État égyptien, en adoptant une définition extrêmement restrictive de l'urbain, tente de se persuader que l'urbanisation ne progresse pas : de 1960 à 2006, le taux d'urbanisation aurait stagné à 43 \% de la population et aurait même baissé depuis 1986. En revanche, les mesures de l'urbanisation qui considèrent les agglomérations de plus de 10000 habitants englobent $82 \%$ de la population (Moriconi, 2011). C'est dans ce cadre urbain qui conjugue densité et diversité que s'inventent les formes d'autonomie et d'auto-organisation sociale rendues indispensables par le désengagement de l'État. L'explosion de l'habitat illégal depuis les années 1980, du fait de l'absence de politiques du logement adaptées aux besoins, en est une bonne illustration.

$\mathrm{Au}$ cœur de cette urbanisation, on trouve une société extrêmement mobile alors que les Égyptiens apparaissent dans l'imaginaire national comme des sédentaires par excellence, captifs des rives du Nil. Cela s'observe tant sur le plan interne avec le développement des transports en commun (microbus, touks-touks ${ }^{12}$ ) qui irriguent les villages plus reculés que sur le plan externe avec le développement massif de la migration internationale vers le Golfe principalement. À l'intérieur de l'Égypte, cette mobilité a tissé un intense réseau de déplacements pendulaires qui se sont substitués aux flux de migration des ruraux vers les villes. Enfin, la mobilité s'organise au bénéfice des villes de province, grandes comme petites, qui continuent de polariser les activités et les services, en répétant à l'échelle locale la polarisation exercée par la région du Grand Caire sur toute l'Égypte.

Cette mobilité des personnes, des biens et des services permet la diversification de l'activité dans toutes les zones habitées, ainsi que la diffusion de nouveaux modes de vie comme celle de l'information. Ce phénomène revêt une grande portée car la mobilité des hommes s'accompagne de celle des idées: les idées neuves ont gagné ainsi le pays profond.

\section{Frustration et génie des jeunes générations}

Parler de la révolution du 25 janvier comme d'une "révolution de la jeunesse », reste, d'un certain point de vue, bien imprécis: ces dizaines de millions de jeunes gens ne peuvent être rangés sous la même étiquette, et il y a autant de jeunesses égyptiennes que de groupes sociaux, culturels, etc. On peut cependant avancer qu'une communauté de sentiments et d'expérience est partagée par ce qu'il convient de désigner comme les «nouvelles générations »: une frustration intense, face aux blocages économiques et sociaux auxquels elles sont confrontées. 
ette frustration est d'autant plus forte que les jeunes générations se sentent dépossédées des promesses associées à l'éducation qu'elles ont reçues, dans l'enseignement primaire ${ }^{13}$ ou supérieur, ${ }^{14}$ puisque même les jeunes diplômés ne trouvent pas d'emploi. La garantie donnée par Nasser d'un emploi dans la fonction publique pour chaque diplômé qui a conduit à l'asphyxie de la fonction publique, a été abandonnée au début des années 1990. L'économie égyptienne, même avec des taux de croissance du PIB supérieurs à $5 \%$ par an dans les années 2000, ne parvient pas à créer chaque année les 750000 emplois nécessaires à la résorption du chômage, du fait d'investissements insuffisants dans les secteurs économiques créateurs d'emplois. Or, le chômage touche massivement les nouveaux entrants sur le marché du travail : en 2005, 92 \% des chômeurs officiellement recensés ${ }^{15}$ sont dans la catégorie des 15 à 29 ans (El-Megharbel, 2008).

Les jeunes générations connaissent donc le chômage (les femmes notamment, qui semblent les premières victimes de la fermeture de l'emploi public) ou entrent dans la vie professionnelle via l'emploi informel. Celui-ci absorbait entre 2000 et 2005, plus de $75 \%$ des nouveaux entrants sur le marché du travail (Assad, 2007). Pour les jeunes générations, étudier et trouver un emploi est devenu difficile, avec pour principale conséquence le recul de l'âge au mariage, institution centrale de la société égyptienne, puisque le mariage nécessite l'achat d'un logement équipé par le mari : de nombreux jeunes ont été obligés d'émigrer pour pouvoir amasser un pécule et acheter un appartement pour loger leur famille. Compte tenu de l'exiguïté de l'œkoumène, les prix du foncier sont très élevés en Égypte. Les jeunes ont alors tendance à acheter ou louer des logements dans les périphéries des villes, bien souvent dans les quartiers informels où ils trouvent un habitat à la mesure de leurs moyens.

Enfin, la remise en question des cadres hérités se double de l'expérience, souvent amère, du quotidien. Les nouvelles générations sont les témoins directs de l'échec de leurs pères : échec des expériences politiques de référence (socialisme ou islamisme radical), verrouillage du système politique par une gérontocratie, échec en termes de développement d'un pays miné par la corruption et la pauvreté. La rupture générationnelle des représentations s'est par ailleurs accompagnée de la perméabilité croissante des jeunes générations au monde extérieur, à la mondialisation culturelle, grâce aux nouveaux médias mais également à l'explosion des mobilités décrites plus haut. Comme l'indique l'anthropologue Arjun Appadurai (1996), dans ce processus de mélange des imaginaires propre à la mondialisation, le monde extérieur devient partie intégrante du monde intérieur de chacun. Les nouvelles générations sont donc « en attente » d'une réelle alternative, et elles sont descendues dans la rue pour aller l'y chercher. Il n'est pas possible actuellement de deviner quelle société va en naître. Mais il est tentant de lire, lors des manifestations de 2011, une traduction politique de cette attente, dont ont rendu compte, en creux, l'absence de référence aux modèles politiques des générations précédentes, à des leaders identifiés, ou encore le rejet de l'autorité et des figures paternelles de tous ordre (celle du raïs la première).

\section{Conclusion : la ville comme lieu de la revolution arabe}

51 Depuis le 11 février, les jeunes révolutionnaires mènent un constant bras de fer avec l'armée. À leurs yeux, le démantèlement du système est trop lent. Ils dénoncent le rétablissement de l'appareil répressif et le pacte conservateur implicite scellé entre l'armée et les Frères musulmans. Pour se faire entendre, ils sont retournés, 
régulièrement, manifester sur la place Tahrir - que l'armée a essayé d'interdire au cours de l'été 2011. Ce faisant, la place Tahrir est devenue à elle seule le symbole de toutes les révolutions arabes: les grandes places de Manama (Bahreïn), Benghazi (Libye) et Homs (Syrie) ont été chacune rebaptisées place Tahrir par les manifestants rendant compte sans doute de la logique commune de ces mouvements révolutionnaires arabes, qui se pensent tous comme des mouvements de libération du peuple face à un pouvoir qui a confisqué l'État à la Nation (Filiu, 2011). L'une des leçons largement oubliée de la révolution égyptienne serait peut-être celle-ci : alors que l'on célèbre les cyber-révolutions arabes et la victoire de l'espace virtuel sur la tyrannie, l'appropriation de l'espace physique et concret des lieux centraux et symboliques de l'histoire des peuples est toujours aussi nécessaire pour combattre l'oppression et fonder une société nouvelle. Dans l'ère inaugurée par le printemps arabe, la géographie a de beaux jours devant elle...

\section{BIBLIOGRAPHIE}

Anderson B., 2006 [1983]. Imagined communities : reflections on the origin and spread of nationalism. Londres, $240 \mathrm{p}$.

Ambrosetti E., 2011. Égypte, l'exception démographique. Paris, Les Editions de l'INED, 255 p.

Amnesty International, 2011. Egypt rises. Killings, Detentions and Torture in the '25 January revolution'. $101 \mathrm{p}$.

Appadurai A., 1996. Modernity at Large: Cultural Dimensions of Globalization. Minneapolis, University of Minnesota Press, 229 p.

Assaad R., 2007. Labor supply, employment and unemployment in the Egyptian economy, 1988-2006. Working Paper $n^{\circ}$ 0701, Cairo, ECES, 46 p.

Badie B., 2011. La revanche des sociétés arabes. Le Monde.Fr, 24 février. http:// abonnes.lemonde.fr/idees/article/2011/02/24/bertrand-badie-les-societes-prennent-leurrevanche-en-tunisie-Égypte-et-libye_1484863_3232.html (Consulté le 13 mai 2011).

Béchir Ayari M., 2011. « Non, les révolutions tunisiennes et égyptiennes ne sont pas des révolutions $2.0 »$. Mouvements, $n^{\circ}$ 65, p. 56-61.

Ben Néfiss S., 2010. Verrouillage autoritaire et mutation générale des rapports enter l'État et la société en Égypte. Confluences Méditerranée, vol. 75, p. 137-150.

Ben Néfissa S., 2009. Égypte : nouvelles dimensions des protestations sociales ». États des résistances dans le Sud -Monde arabe 2010, Louvain-la-Neuve, Paris, Centre tricontinental, Éd. Syllepse. http://www.cetri.be/spip.php ?article1471\&lang =fr (Consulté le 11 mai 2011).

Beinin J., 2011. Egypt: Revolution or Military Coup? Avicenna, The Stanford Journal on Muslim Affairs, Stanford University, vol. 1, p. 6-10.

Bruyas F., 2007. Mégaprojets » nationaux et dynamiques identitaires locales : Le cas de Port-Saïd et des autres villes du canal de Suez. In Denis E. (dir.), Villes et urbanisation des provinces égyptiennes. Vers l'écoumènopolis ?, Paris, Le Caire, Karthala, CEDEJ, p. 291-328. 
Clément F., Moisseron J.-Y., 2007. Changements visibles ou invisibles : la question de l'émergence de l'économie égyptienne? Politique africaine, $\mathrm{n}^{\circ} 108$, Karthala, $220 \mathrm{p}$.

Courbage Y., Todd E., 2007. Le rendez-vous des civilisations. Paris, Le Seuil, La république des idées, $184 \mathrm{p}$.

Daoud A., 2011. « La révolution tunisienne de janvier 2011 : une lecture par les déséquilibres du territoire », EchoGéo [En ligne], Sur le vif 2011, mis en ligne le 23 septembre 2011, consulté le 02 octobre 2011. URL : http://echogeo.revues.org/12612

Denis E., 2011. La financiarisation du foncier observée à partir des métropoles égyptiennes et indiennes. Revue Tiers Monde, dossier « Dynamiques foncières dans les villes du Sud », nº 206.

Denis E., Vignal L., 2003. Dimensions nouvelles de la métropolisation dans le Monde arabe : le cas du Caire. Les échelles de la métropolisation en Égypte, entre sur-polarisation et redistribution ». Les Cahiers de la Méditerranée, p. 119-166.

El-Megharbel N., 2008. The impact of recent macro and labor market policies on job creation in Egypt. In Kheir El-Din H. (ed.), The Egyptian Economy, Current Challenges and Future Prospects, AUC Press, Cairo, 338 p.

Fargues P., 2000. Générations arabes, L'alchimie du nombre. Fayard, Paris, 349 p.

Filiu J.-P., 2011, La révolution arabe, Dix leçons sur le soulèvement démocratique. Fayard, Paris, $251 \mathrm{p}$.

Kienle E., 2001. A grand delusion: democracy and economic reform in Egypt. London, I.B. Tauris, 274 p. Moriconi-Ebrard F., 2011. Égypte. Tableau de bord de l'urbanisation 1950-2020, site e-geopolis, http://e-geopolis.eu/menapolis/Tableau_bord_Égypte_final.pdf (consulté le 13 mai 2011).

Pagès D., Vignal L. 1998. Formes et espaces de la mondialisation en Égypte, Revue Géographique de Lyon, vol. 73, p. 247-258.

Pagès-El Karoui D., 2008. Villes du delta du Nil : Tantâ, Mahalla, Mansûra, cités de la densité, Paris, Le Caire, Editions Karthala, CEDEJ, 357 p.

Sanmartin O., 2010. La péninsule du Sinaï : « espace projet », territoire sous tensions. Confluences Méditerranée, vol 4, nº 75, p. 107-120.

Vignal L., 2010a. The new territories of tourism in Egypt : a local-global frontier ? Cybergeo : European Journal of Geography. http://cybergeo.revues.org/23324 [Consulté : 5 juillet 2011].

Vignal L., 2010 b. Reforming Egypt ? From the Association Agreement to the European Neighbourhood Policy, fifteen years of EU/Egypt cooperation, Working paper, RAMSES European Network of Excellence.

\section{NOTES}

1. Agglomération de plus de 10000 habitants.

2. Ministry of Communications and Information, Egypt, ICT Indicators in Brief, Monthly Report February 2011. http://www.mcit.gov.eg/Upcont/Documents/ICTinBriefFeb2011-E.pdf, page 2. (Consulté le 12 juin 2011).

3. http://www.socialbakers.com, (consulté le 28 avril 2011).

4. Unité de découpage administratif inférieure au gouvernorat.

5. Nous verrons cependant que la notion même de village doit être comprise avec précaution dans un pays massivement urbanisé (cf. infra). 
6. Base statistique, CNUCED, 2011.

7. Central Bank of Egypt, Economic Review, Vol. 50, N 4, 2009/2010. http://www.cbe.org.eg/ public/EconomicVol50N4.pdf (consulté le 23 juin 2010)

8. Central Bank of Egypt, Economic Review, ibid.

9. Base statistique, Banque Mondiale, 2011.

10. Egypt Labor Market Panel Survey, 2006, disponible sur le site de l'ERF (Economic Research Forum, Le Caire) : www.erf.org.eg

11. La division administrative de l'Égypte s'effectue entre des gouvernorats entièrement urbains comme ceux du Caire ou d'Alexandrie et des gouvernorats dits ruraux qui comportent un secteur urbain, les villes, chefs-lieux de gouvernorat et de district, et un secteur rural.

12. Il s'agit de triporteurs importés d'Inde, ou autorickshaws. Ces mini-taxis qui ne peuvent prendre que deux personnes, plus le chauffeur, circulent notamment dans les petites villes de province et les bourgs périurbains.

13. La politique d'éducation massive fut engagée sous Nasser afin d'offrir une éducation gratuite pour tous. Jusqu'à $25 \%$ du budget national est alors consacré à l'éducation. Mais, au milieu des années 1970, la massification de l'enseignement imposée par la croissance démographique conduit à un système à plusieurs vitesses, fondé sur des cours particuliers "obligatoires » parallèles dans l'école publique qui grèvent sévèrement le budget des familles et le recours à l'enseignement privé pour les familles aisées.

14. La politique universitaire engagée sous Nasser a formellement porté ses fruits : on compte aujourd'hui une vingtaine d'universités publiques en Égypte, qui formaient, en 2003, plus de 1,5 millions d'étudiants: la moitié au Caire, l'autre moitié dans les provinces, sans compter la multiplication partout dans le pays des instituts techniques censés délivrer des formations professionalisantes.

15. Les définitions officielles du chômage ne correspondent guère à la réalité d'un pays qui compte au moins 9 millions de travailleurs dans le secteur informel.

\section{RÉSUMÉS}

Cet article identifie les racines structurelles de la révolution égyptienne du 25 janvier 2011. Si le blocage du système politique et les promesses de développement non tenues de la libéralisation économique ont fait le lit de la contestation, les profondes mutations de la société égyptienne au cours des 50 dernières années - baisse de la fécondité, essor de l'éducation, urbanisation massive - sont autant de causes profondes qui expliquent le soulèvement populaire et le départ d'Hosni Moubarak.

This paper explores the structural roots of the 25th January Egyptian revolution. The stalemate of the political system and the deceptive effects of an economic liberalization with little trickledown effect explain the run-up to the revolution. However, deep social changes (the decline in birth rates, the rise of literacy, massive urbanization) are part of the background to the popular uprising and the exit of Mubarak. 


\section{INDEX}

Mots-clés : révolution, Egypte, libéralisation, villes, urbanisation, transition démographique, TIC

Keywords : Egypt, liberalization, cities, demographic transition, ICT

\section{AUTEURS}

\section{DELPHINE PAGÈS-EL KAROUI}

Delphine Pagès-El Karoui est Maître de conférences en géographie à l'INALCO (Institut National des Langues et Civilisations Orientales).dpages@inalco.fr . Elle a publié récemment :

- Pagès-El Karoui D., 2010. Shisha Cafés in France: Reinventing the Oriental Dream. From Exoticism to Beurs Integration. Die Erde, $\mathrm{n}^{\circ} 141$ (1-2), Special issue New geographies of migration, p. 31-63.

- Pagès-El Karoui D., 2008. Villes du Delta du Nil. Tantâ, Mahalla, Mansûra, cités de la densité. Paris, Karthala, 357 p.

- Bennafla K., Pagès-El Karoui D., Sanmartin O., 2007. Géopolitique du Maghreb et du Moyen-Orient. Paris, SEDES, $230 \mathrm{p}$.

\section{LEILA VIGNAL}

Leïla Vignal est Maître de conférences en géographie à l'Université de Rennes-2/ Chaire d'excellence CNRS. leila.vignal@univ-rennes2.fr. Elle a publié récemment :

- Vignal L., 2010. The new territories of tourism in Egypt: a local-global frontier?, Cybergeo : European Journal of Geography, article 509, mis en ligne le 29 octobre 2010, modifié le 17 novembre 2010. URL : http://cybergeo.revues.org/23324

- Vignal L, 2011. Jours tranquilles à Damas. Quelques aperçus sur la révolte syrienne à la mi-mai 2011. Revue Esprit, Juin 2011, Paris, p. 94-102.

- Vignal L., 2011. Beyrouth, de la boutique au shopping mall : Dynamiques métropolitaines et nouvelle géoéconomie au Moyen-Orient. In Marchés, boutiques, souks et mall : formes sociales et spatiales de l'échange marchand en Méditerranée. Mermier F., Péraldi M. (dirs.), Editions de la Découverte, Paris, p. 279-302. 